\title{
The Efficacy And Safety Of Apatinib Treatment For Patients With Metastatic Or Recurrent Cervical Cancer: A Retrospective Study
}

This article was published in the following Dove Press journal: Drug Design, Development and Therapy

\author{
Jiangtao $\mathrm{Yu} \mathbb{1}^{1,2, *}$ \\ Ziwen $\mathrm{Xu}^{3, *}$ \\ Anyang $\mathrm{Li}^{2}$ \\ Jindi Zhang $\mathbb{D}^{2}$ \\ Yi Wang ${ }^{4}$ \\ Hongqin Zhao ${ }^{2}$ \\ Haiyan Zhu ${ }^{1,2}$
}

'Department of Gynecology, Shanghai First Maternity and Infant Hospital, Tongji University School of Medicine, Shanghai 200 I26, People's Republic of China; ${ }^{2}$ Department of Gynecology, The First Affiliated Hospital of Wenzhou Medical University, Wenzhou 325000, People's Republic of China; ${ }^{3}$ Department of Medicine, Wenzhou Medical University, Wenzhou 325015, People's Republic of China; ${ }^{4}$ Department of Gynecology, Wenzhou Oncology Hospital, Wenzhou 325000, People's Republic of China

*These authors contributed equally to this work

\begin{abstract}
Background: This study was performed to assess the efficacy and safety of apatinib in patients with metastatic or recurrent cervical cancer.

Methods: Twenty-six patients with metastatic or recurrent cervical cancer and treated with apatinib until progressive disease or unacceptable toxicity were included in this multicenter, retrospective, observational study from January 2016 to April 2018. The primary end point was progression free survival (PFS). Secondary end points included overall survival (OS), objective response rate (ORR), disease control rate (DCR), and toxicity. Toxicities were assessed according to Common Terminology Criteria for Adverse Events.
\end{abstract}

Results: A total of 26 metastatic or recurrent cervical cancer patients were enrolled in this study. No complete response (CR) occurred, 4 patients (15.4\%) showed partial response (PR), 11 patients $(42.3 \%)$ had stable disease (SD), and 11 patients $(42.3 \%)$ had progressive disease (PD), with DCR of $57.7 \%$ and ORR of $15.4 \%$. Median progression-free survival (PFS) was 3.0 months (95\% confidence interval [CI]: 0-6.3 months) and overall survival (OS) was 7.0 months (95\% CI: 5.1-8.9 months) respectively. The most common adverse effects were hand-foot syndrome (50.0\%), secondary hypertension (26.9\%) and fatigue $(26.9 \%)$. Three patients discontinued treatment due to grade 3 toxicities (one case for hand-foot syndrome, two cases for diarrhea) and 6 patients required dose reduction because of adverse effects.

Conclusion: Apatinib seems active in heavily-pretreated metastatic or recurrent cervical cancer. The adverse effects were moderate but manageable.

Keywords: epidermal growth factor receptor, apatinib, cervical cancer, metastatic or recurrent, efficacy

\section{Introduction}

Cervical cancer is the fourth most common malignancy in women worldwide. ${ }^{1}$ Although improved screening and human papillomavirus immunization, approximately 98,900 newly are diagnosed and 30,500 women still die from this disease each year in China. ${ }^{2}$ The current standard treatment of cervical cancer is surgery in the early stages and concurrent cisplatin chemotherapy and pelvic radiation in locally advanced lesions. However, patients with recurrent or metastatic cancers have a poor prognosis with limited treatment options. Thus, it is urgent to discover novel active drugs against this disease.

Apatinib is an oral, highly potent tyrosine-kinase inhibitor targeting vascular endothelial growth factor receptor 2 (VEGFR-2). ${ }^{3}$ Currently, apatinib has been demonstrated efficacy in various cancer types, including ovarian cancer, ${ }^{4,5}$ breast cancer, ${ }^{6}$
Correspondence: Haiyan Zhu Department of Gynecology, Shanghai First Maternity and Infant Hospital, Tongji University School of Medicine, Shanghai 200 I26, People's Republic of China Tel +86 I37584652255

Email zhuhaiyandoc@sina.com 
gastric cancer, ${ }^{7,8}$ lung cancer ${ }^{9}$ and liver cancer. ${ }^{10}$ Preclinical data showed that apatinib markedly induced apoptosis and G1-phase arrest, suppressed cell growth, and decreased colony formation ability of cervical cancer cells, indicating apatinib had the potential anti-tumor effects in cervical cancer. ${ }^{11}$ However, the treatment of apatinib in patients with cervical cancer is still unclear. Thus, this retrospective study was to evaluate the efficacy and safety of apatinib in metastatic or recurrent cervical cancer in China.

\section{Methods}

\section{Patients}

A total of 26 metastatic or recurrent cervical cancer patients who administered aptinib between January 2016 and April 2018 at the First Affiliated Hospital of Wenzhou Medical University and Wenzhou oncology Hospital were enrolled in this study. Eligibility criteria were as followed: age $\geq 18$ years; pathologically confirmed as cervical cancer; at least one measurable lesion according to Response Evaluation Criteria in Solid Tumors (RECIST) version $1.1 ;^{12}$ definition of metastasis or recurrence: demonstration of measurable tumor according to CT/MRI/PET-CT. The study was approved by the Ethics Committee of the First Affiliated Hospital of Wenzhou Medical University and informed consent was obtained from each patient before the initiation of the study.

\section{Treatment}

Apatinib was provided as tablets to be administered orally daily. Patients administered aptinib at 250 or $500 \mathrm{mg}$ daily until disease progression or unacceptable toxicity. The dosage of the apatinib was determined by the attending physician based on the patient's age, body weight, general status, and tolerance. Performance status, blood pressure, complete blood count, urine, liver and kidney function were monitored during treatment. Four weeks were defined as one cycle. Only patients who had finished at least one cycle apatinib therapy and evaluated the efficacy were included in this study (Two patients were excluded because they only administered apatinib for 1-2 weeks. One patient stopped the treatment because the treatment was beyond her financial burden, the other patient stopped the treatment because of family reasons, thus, a total of 26 patients were enrolled in the current study.).

\section{Efficacy And Safety Assessments}

The efficacy evaluation was evaluated according to the RECIST 1.1 criteria, ${ }^{12}$ including complete response (CR), partial response (PR), stable disease (SD), and progressive disease (PD). The objective response rate (ORR) was defined as the proportion of eligible patients who achieved a confirmed CR or PR. Disease control rate (DCR) was defined as the proportion of patients who achieved CR, PR, and SD for at least 8 weeks. Progression-free survival (PFS) was defined as the time from initiation of apatinib to the date of disease progression or death, whichever occurred first. Overall survival (OS) was defined as the time from initiation of apatinib to death or the last follow-up visit. CT/MRI/PET-CT was done after 2-3 months of treatment. The deadline for the follow-up time was October 1, 2018.

Apatinib related toxicities were evaluated and graded according to the National Cancer Institute. Toxicities were assessed according to the Common Terminology Criteria for Adverse Events (CTCAE). ${ }^{13}$

\section{Statistical Analysis}

Statistical analyses were performed by SPSS version 24.0 (IBM, Chicago, IL). Continuous data were expressed as the median (range) and categorical variables were presented as percentages. Median PFS and OS were calculated using the Kaplan-Meier method.

\section{Results}

\section{Patient Characteristics}

A total of 26 patients with cervical cancer were included in this retrospective study. Patient characteristics at baseline are summarized in Table 1. The median age was 58 years (range $=36-72$ years). Twenty-three patients were diagnosed as squamous cervical cancer, two patients were adenocarcinoma and one patient was adenosquamous carcinoma. All 26 individuals were confirmed as patients with metastatic or recurrent disease. The most common sites of metastatic disease were lymph nodes (61.5\%), lung (34.6\%) and bone (26.9\%). 15 (57.7\%) patients presented with at least one site of visceral metastasis. In addition, 18 of 26 patients (69.2\%) were pretreated (defined as the treatment after the confirmation of metastasis or recurrence) with chemoradiotherapy, 4 patients with chemotherapy and 2 patients with radiotherapy. Only two patients received no chemotherapy or radiotherapy after confirmed as metastatic or recurrent disease. None of the patients was pretreated with other antiangiogenic therapy, such as bevacizumab.

\section{Safety}

All 26 patients received at least one dose of apatinib and were included in survival and safety analyses. Finally, 21 patients 
Table I The Baseline Patients' Characteristics

\begin{tabular}{|l|l|}
\hline Characteristics & Results \\
\hline Age (years) & $58(36-72)$ \\
\hline Histology, n (\%) & \\
Squamous cell carcinoma & $23(88.5 \%)$ \\
Adenocarcinoma & $2(7.7 \%)$ \\
Adenosquamous carcinoma & $1(3.8 \%)$ \\
\hline Differentiation & \\
Unknown & $14(53.8 \%)$ \\
I-II & $6(23.1 \%)$ \\
III & $6(23.1 \%)$ \\
\hline Metastatic or recurrent sites, $n(\%)$ & \\
Lymph nodes & $16(61.5 \%)$ \\
Lung & $9(34.6 \%)$ \\
Bone & $7(26.9 \%)$ \\
Liver & $5(19.2 \%)$ \\
Pelvic & $5(19.2 \%)$ \\
Brain & $4(15.4 \%$ \\
Rectum & $1(3.8 \%)$ \\
\hline Visceral metastasis, n(\%) & \\
Yes & $15(57.7 \%)$ \\
No & $11(42.3 \%)$ \\
\hline Prior therapy *n (\%) & \\
Chemo+ Radiotherapy & $18(69.2 \%)$ \\
Chemotherapy & $4(15.4 \%)$ \\
Radiotherapy & $2(7.7 \%)$ \\
No & $2(7.7 \%)$ \\
\hline
\end{tabular}

Note: *Prior treatment after the confirmation of metastasis or recurrence. Abbreviation: $n$, number of patients.

(80.8\%) started from $500 \mathrm{mg}$ daily, in which 6 patients experienced dose reduction to $250 \mathrm{mg}$ because of intolerable toxicity, and the other 5 patients $(19.2 \%)$ started from a dose of $250 \mathrm{mg}$ daily. Three patients discontinued treatment due to intolerable toxicity, which was grade 3 hand-foot syndrome and grade 3 diarrhea.

Detailed toxicities encountered in the study are presented in Table 2. Most toxicities were mild (grade 1 to 2 ) and manageable. Only three patients occurred grade 3 adverse effects. No treatment-related death was documented during the drug administration. Four patients suffer no obvious toxicity. Hand-foot syndrome was the most common toxicity, in which one patient suffered grade 3 and discontinued treatment. Secondary hypertension occurred in 7 patients $(26.9 \%)$. Seven patients $(26.9 \%)$ developed fatigue in grade $1-2$. Two patients suffered grade 3 diarrhea and caused discontinued treatment. Other rare side effects were anorexia, hematologic toxicities, nausea, and liver dysfunction, mainly as grade 1 to 2 .

\section{Efficacy}

Median follow-up time was 5.0 months (range, 3 to 24 months) and 16 of 26 patients were dead. Among the 26 patients, there were four with PR (15.4\%), 11 with SD $(42.3 \%)$ and 11 patients $(42.3 \%)$ with PD. However, no CR occurred. The overall response rate was $15.4 \%$. The disease control rate (CR plus PR plus SD) was 57.7\%. Additionally, the median disease control duration was 5 months (range $=3-24)$, and 5 out of 15 patients $(33.3 \%)$ had a disease control duration $\geq 6$ months.

Median PFS and OS were 3.0 (95\% CI 0-6.3) and 7.0 (95\% CI 5.1-8.9) months, respectively (Figure 1).

\section{Discussion}

Women with metastatic or recurrent cervical cancer are still incurable with a $15-20 \% 1$-year-OS. ${ }^{14}$ Cisplatin-based chemotherapy palliates symptoms and prolongs PFS of these patients, and thus, represents the standard of treatment for this population. ${ }^{15,16}$ However, the low response rate and relatively short OS are disappointing. The presented study evaluated the efficacy and safety of apatinib among patients with advanced cervical cancer. Our results showed a $57.7 \%$ DCR and a median PFS of 3 months. Interestingly, our study showed a high rate of DCR and a long period of PFS, however, CR and PR were $0 \%$ and $15.4 \%$, respectively. Current viewpoints suggested that the potential benefit from novel targeted agents was mediated through disease stabilization processes rather than tumor shrinkage. ${ }^{17-19}$ Therefore, the primary end point of this study was determined as PFS. Accordingly, apatinib seemed to be efficacious for patients with recurrent or metastatic cervical cancer.

Angiogenesis is a critical process for cancer development and metastasis, including cervical cancer. Target angiogenic pathway is shown to be an effective strategy for controlling cervical cancer progress, especially for advanced cervical cancer. ${ }^{20-22}$ Bevacizumab, a recombinant humanized monoclonal antibody directed against VEGF-A, was the first clinically available anti-angiogenic agent. On August 14, 2014, this targeted agent was approved in the treatment of persistent, recurrent, or metastatic cervical cancer with cisplatin and paclitaxel chemotherapies. According to a recent review paper, the response rate of bevacizumab ranged from $34-48 \%$, and the PFS presented as 3.4-8.2m for recurrent, persistent, or metastatic cervical cancer. ${ }^{23}$ The largest trial (GOG 240) reported a prolonged OS by 3.7 months in patients with 
Table 2 Treatment-Related Toxicities

\begin{tabular}{|l|l|l|l|l|l|}
\hline Adverse Event & GradeI $\mathbf{n}$ & Grade2 $\mathbf{n}$ & Grade3 $\mathbf{n}$ & Grade4 $\mathbf{~}$ & Total $\mathbf{n}(\%)$ \\
\hline Hand and foot syndrome & 4 & 8 & 1 & 0 & $13(50.0 \%)$ \\
Secondary hypertension & $\mathrm{I}$ & 6 & 0 & 0 & $7(26.9 \%)$ \\
Fatigue & 3 & 4 & 0 & 0 & $7(26.9 \%)$ \\
Anorexia & $\mathrm{I}$ & 5 & 0 & 0 & $6(23.1 \%)$ \\
Neutropenia & 0 & 4 & 0 & 0 & $4(15.4 \%)$ \\
Thrombocytopenia & 0 & 4 & 2 & 0 & $4(15.4 \%)$ \\
Diarrhea & 0 & 2 & 0 & 0 & $4(15.4 \%)$ \\
Liver dysfunction & $\mathrm{I}$ & 1 & 0 & $2(7.7 \%)$ \\
Nausea & 0 & 2 & 0 & $2(7.7 \%)$ \\
\hline
\end{tabular}

Abbreviation: n, number of patients.
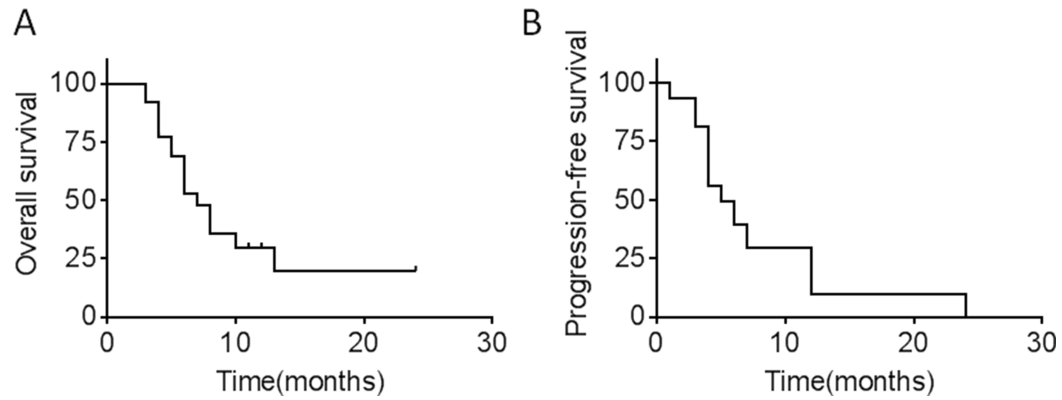

Figure I (A) OS in 26 patients with metastatic or recurrent cervical cancer after apatinib treatment. (B) PFS in 26 patients with metastatic or recurrent cervical cancer after apatinib treatment.

recurrent, persistent, or metastatic cervical cancer by bevacizumab combined with standard chemotherapy. ${ }^{20}$ Overall, the use of bevacizumab in cervical cancer showed effective and safe, but with high costs. ${ }^{23}$ Previous studies also evaluated alternative antiangiogenic strategies, including cetuximab and sunitinib. Cetuximab(C226), an antibody that inhibits EGFR activity, did not indicate additional benefit beyond cisplatin therapy alone along with adequately tolerated in advanced, recurrent, and previously treated cervical cancers. ${ }^{21}$ Along similar lines, sunitinib malate, multitargeted TKI, showed no objective responses in pretreated patients with locally advanced or metastatic cervical cancer. $^{24}$ Compared with previously reported anti-VEGF drugs, apatinib showed a relative high disease control rate and a long PFS. Further prospective studies with larger sample size need to confirm this observation.

The main challenge regarding antiangiogenic effects in clinical practice is drug resistance and response rate across patients. Thus, it is important to select relative appropriate patients. It was reported that bevacizumab seemed to be less effective in patients with adenocarcinoma than in those with squamous cancer in a Phase III Gynecologic Oncology
Group (GOG 240) study. $^{20}$ Our study only enrolled two cases of adenocarcinoma, in which one presented SD and another case showed PR. Considering the small size in the current study, inadequate data cannot make a definitive judgment regarding histology. In addition, it was widely reported that single-agent activity of angiogenesis inhibitors was limited and combination with standard chemotherapy was recommended. Actually, most the previous studies presented bevacizumab efficiency combined with chemotherapy. ${ }^{23}$ Only in GOG227C study, single bevacizumab showed active in treating recurrent $\mathrm{CC}$ with a OS $7.3 \mathrm{~m}$ and PFS $3.4 \mathrm{~m} .{ }^{15} \mathrm{~A}$ probable explanation for these findings is antiangiogenic therapies could create or remodel an environment suitable for normalization of the stable vascular endothelial cells, thus, result in increased tumor uptake of chemotherapy. ${ }^{25,26}$ In the presented study, the majority of patients previously received platinum-based chemotherapy after diagnosed as metastasis or recurrence. Only two patients refused chemotherapy and selected single apatinib. Interestingly, both of these two patients showed PD. Thus, apatinib may be a promising targeted agent for the heavily-pretreated advanced cervical cancer. We would suggest use apatinib in combination with one or more traditional chemotherapeutic drugs. 
Hypertension, proteinuria, and hand-foot syndrome are the most common adverse events related to anti-angiogenic agents. ${ }^{27}$ Our current study showed the most frequently observed side effect were hand-foot syndrome, hypertension and fatigue, which were generally consistent with previously reported in ovarian cancer and breast cancer. ${ }^{4,17}$ Interestingly, hand-foot syndrome is the most frequently observed side effect in the current study, in addition, one patient discontinued treatment because of grade 3 hand-foot syndrome. The mechanism of hand-foot syndrome is probably due to the damage of the capillary endothelium in hands and feet as well as keratinocyte apoptosis and inflammation by inhibiting VEGF. $^{27}$ Clinically, the management of hand-foot syndrome has always been a tough thing. One patient in our study discontinued treatment because of grade 4 hand-foot syndrome. Thus, prophylactic measures, including moisturization for hand and foot, protection of pressure-sensitive areas, herbal medicine immersion and supplementation of multi-vitamin should be considered. Overall, in the current study, apatinib by a dosage of $250-500 \mathrm{mg}$ daily is tolerable for most heavily pretreated advanced cervical cancer. Furthermore, no unusual toxicities were identified among all the 26 patients. Thus, apatinib seemed not only active in treating metastasis or recurrent cervical cancer but also safe.

\section{Conclusion}

This study demonstrated that apatinib was potentially an effective drug for patients with heavily pretreated metastasis or recurrent cervical cancers. The toxicities were moderated but manageable. Thus, patients with advanced cervical cancer can tolerate and benefit from apatinib therapy, which makes apatinib therapy a promising option.

\section{Abbreviations}

VEGFR-2, vascular endothelial growth factor receptor 2; RECIST, Response Evaluation Criteria in Solid Tumors; $\mathrm{CR}$, complete response; $\mathrm{PR}$, partial response; SD, stable disease; PD, progressive disease; ORR, objective response rate; DCR, disease control rate; PFS, progression-free survival; OS, overall survival; CTCAE, common terminology criteria for adverse events.

\section{Ethical Approval}

This study has been approved by The First Hospital of Wenzhou Medical University's ethics committee and has been performed according to the ethical standards laid down in the 1964 Declaration of Helsinki.

\section{Informed Consent}

Informed consent was written by all individual participants included in the study.

\section{Funding}

This work was supported by grants from the Natural Science Foundation of Zhejiang Province (No. LY19H160021), Technology Development Funds of Wenzhou City (Y20150046) and the Young Talent Fund of the First Affiliated Hospital of Wenzhou Medical University.

\section{Disclosure}

The authors report no conflicts of interest in this work.

\section{References}

1. Torre LA, Bray F, Siegel RL, Ferlay J, Lortet-Tieulent J, Jemal A. Global cancer statistics, 2012. CA Cancer J Clin. 2015;65:87-108. doi: $10.3322 /$ caac. 21262

2. Chen W, Zheng R, Baade PD, et al. Cancer statistics in China, 2015. CA Cancer J Clin. 2016;66:115-132. doi:10.3322/caac.21338

3. Ding J, Chen X, Gao Z, et al. Metabolism and pharmacokinetics of novel selective vascular endothelial growth factor receptor-2 inhibitor apatinib in humans. Drug Metab Dispos. 2013;41:1195-1210. doi:10.1124/dmd.112.050310

4. Miao M, Deng G, Luo S, et al. A phase II study of apatinib in patients with recurrent epithelial ovarian cancer. Gynecol Oncol. 2018;148:286-290. doi:10.1016/j.ygyno.2017.12.013

5. Lan CY, Wang Y, Xiong Y, et al. Apatinib combined with oral etoposide in patients with platinum-resistant or platinum-refractory ovarian cancer (AEROC): a phase 2, single-arm, prospective study. Lancet Oncol. 2018. doi:10.1016/S1470-2045(18)30349-8

6. Hu X, Zhang J, Xu B, et al. Multicenter phase II study of apatinib, a novel VEGFR inhibitor in heavily pretreated patients with metastatic triple-negative breast cancer. Int $J$ Cancer. 2014;135:1961-1969. doi:10.1002/ijc. 28829

7. Zhang Y, Han C, Li J, et al. Efficacy and safety for Apatinib treatment in advanced gastric cancer: a real world study. Sci Rep. 2017;7:13208. doi:10.1038/s41598-017-13192-8

8. Brower V. Apatinib in treatment of refractory gastric cancer. Lancet Oncol. 2016;17:e137. doi:10.1016/S1470-2045(16)00138-8

9. Xu J, Liu X, Yang S, Zhang X, Shi Y. Apatinib plus icotinib in treating advanced non-small cell lung cancer after icotinib treatment failure: a retrospective study. Onco Targets Ther. 2017;10:49894995. doi:10.2147/OTT.S142686

10. Zhen L, Jiali C, Yong F, Han X, Hongming P, Weidong H. The efficacy and safety of apatinib treatment for patients with unresectable or relapsed liver cancer: a retrospective study. J Cancer. 2018;9:2773-2777. doi:10.7150/jca.26376

11. Qiu H, Li J, Liu Q, Tang M, Wang Y. Apatinib, a novel tyrosine kinase inhibitor, suppresses tumor growth in cervical cancer and synergizes with Paclitaxel. Cell Cycle. 2018;1-10.

12. Eisenhauer EA, Therasse P, Bogaerts J, et al. New response evaluation criteria in solid tumours: revised RECIST guideline (version 1.1). Eur J Cancer. 2009;45:228-247. doi:10.1016/j.ejca.2008.10.026

13. Trotti A, Colevas AD, Setser A, et al. CTCAE v3.0: development of a comprehensive grading system for the adverse effects of cancer treatment. Semin Radiat Oncol. 2003;13:176-181. doi:10.1016/ S1053-4296(03)00031-6 
14. Eskander RN, Tewari KS. Chemotherapy in the treatment of metastatic, persistent, and recurrent cervical cancer. Curr Opin Obstet Gynecol. 2014;26:314-321. doi:10.1097/GCO.0000000000000042

15. Monk BJ, Sill MW, McMeekin DS, et al. Phase III trial of four cisplatincontaining doublet combinations in stage IVB, recurrent, or persistent cervical carcinoma: a Gynecologic Oncology Group study. J Clin Oncol. 2009;27:4649-4655. doi:10.1200/JCO.2009.21.8909

16. Tewari KS, Monk BJ. Gynecologic oncology group trials of chemotherapy for metastatic and recurrent cervical cancer. Curr Oncol Rep. 2005;7:419-434.

17. $\mathrm{Hu} \mathrm{X,} \mathrm{Cao} \mathrm{J,} \mathrm{Hu} \mathrm{W,} \mathrm{et} \mathrm{al.} \mathrm{Multicenter} \mathrm{phase} \mathrm{II} \mathrm{study} \mathrm{of} \mathrm{apatinib} \mathrm{in}$ non-triple-negative metastatic breast cancer. BMC Cancer. 2014;14:820. doi:10.1186/1471-2407-14-820

18. Llovet JM, Ricci S, Mazzaferro V, et al. Sorafenib in advanced hepatocellular carcinoma. $N$ Engl J Med. 2008;359:378-390. doi:10.1056/NEJMoa0708857

19. Escudier B, Eisen T, Stadler WM, et al. Sorafenib in advanced clearcell renal-cell carcinoma. $N$ Engl J Med. 2007;356:125-134. doi:10.1056/NEJMoa060655

20. Tewari KS, Sill MW, Long HJ 3rd, et al. Improved survival with bevacizumab in advanced cervical cancer. $N$ Engl J Med. 2014;370:734-743. doi:10.1056/NEJMoa1309748

21. Farley J, Sill MW, Birrer M, et al. Phase II study of cisplatin plus cetuximab in advanced, recurrent, and previously treated cancers of the cervix and evaluation of epidermal growth factor receptor immunohistochemical expression: a Gynecologic Oncology Group study. Gynecol Oncol. 2011;121:303-308. doi:10.1016/j.ygyno. 2011.01.030
22. Monk BJ, Sill MW, Burger RA, Gray HJ, Buekers TE, Roman LD. Phase II trial of bevacizumab in the treatment of persistent or recurrent squamous cell carcinoma of the cervix: a gynecologic oncology group study. J Clin Oncol. 2009;27:1069-1074. doi:10.1200/ JCO.2008.18.9043

23. Bizzarri N, Ghirardi V, Alessandri F, et al. Bevacizumab for the treatment of cervical cancer. Expert Opin Biol Ther. 2016;16:407419. doi:10.1517/14712598.2016.1145208

24. Mackay HJ, Tinker A, Winquist E, et al. A phase II study of sunitinib in patients with locally advanced or metastatic cervical carcinoma: NCIC CTG trial IND.184. Gynecol Oncol. 2010;116:163-167. doi:10.1016/j.ygyno.2009.08.012

25. Tong RT, Boucher Y, Kozin SV, Winkler F, Hicklin DJ, Jain RK. Vascular normalization by vascular endothelial growth factor receptor 2 blockade induces a pressure gradient across the vasculature and improves drug penetration in tumors. Cancer Res. 2004;64:3731-3736. doi:10.1158/0008-5472.CAN-04-0074

26. Carmeliet P, Jain RK. Principles and mechanisms of vessel normalization for cancer and other angiogenic diseases. Nat Rev Drug Discov. 2011;10:417-427. doi:10.1038/nrd3455

27. Schmidinger M. Understanding and managing toxicities of vascular endothelial growth factor (VEGF) inhibitors. EJC Suppl. 2013;11:172-191. doi:10.1016/j.ejcsup.2013.07.016

\section{Publish your work in this journal}

Drug Design, Development and Therapy is an international, peerreviewed open-access journal that spans the spectrum of drug design and development through to clinical applications. Clinical outcomes, patient safety, and programs for the development and effective, safe, and sustained use of medicines are a feature of the journal, which has also been accepted for indexing on PubMed Central. The manuscript management system is completely online and includes a very quick and fair peer-review system, which is all easy to use. Visit http://www. dovepress.com/testimonials.php to read real quotes from published authors. 